\title{
Atmospheric influence on a laser beam observed on the OICETS - ARTEMIS communication demonstration link
}

\author{
A. Löscher \\ ESA/ESTEC, Keplerlaan 1, 2200 AG Noordwijk, The Netherlands \\ Received: 18 March 2010 - Published in Atmos. Meas. Tech. Discuss.: 10 May 2010 \\ Revised: 26 July 2010 - Accepted: 20 August 2010 - Published: 14 September 2010
}

\begin{abstract}
In 2006 bi-directional optical inter-satellite communication experiments were conducted between the Japan Aerospace Exploration Agency (JAXA) Optical Inter-orbit Communications Engineering Test Satellite (OICETS) and the European Space Agency (ESA) multi-purpose telecommunications and technology demonstration satellite (Advanced Relay and Technology MISsion) ARTEMIS. On 5 April 2006, an experiment was successfully carried out by maintaining the inter-satellite link during OICETS's setting behind the Earth limb until the signal was lost. This setup resembles an occultation observation where the influence of Earth's atmosphere is evident in the power fluctuations recorded at ARTEMIS's (and OICETS's) receiver. These fluctuations do not exist or are at a low level at a link path above the atmosphere and steadily increase as OICETS sets behind the horizon until the tracking of the signal is lost. This specific experiment was performed only once since atmospheric science was not the goal of this demonstration. Nevertheless, this kind of data, if available more frequently in future, can help to study atmospheric turbulence and validate models. The data present here were recorded at ARTEMIS.
\end{abstract}

\section{Introduction}

Free space laser links have been studied in the context of terrestrial wireless communication to establish performance envelopes and design guidelines (Henninger and Wilfert, 2010). During the last years the capability to employ this technique for establishing communication links between satellites and satellite-to-ground was developed in Europe. One reason for those developments is the growing amount of data generated by Earth Observation Satellites which reached

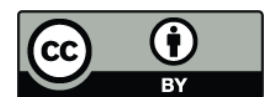

Correspondence to: A. Löscher (armin.loescher@esa.int) the limits concerning the capacity of X-band downlink systems. One possible solution would be the use of optical terminals, increasing the bandwidth significantly, in a direct Earth observation satellite-to-ground or Earth observation satellite-to-relay-satellites-to-ground configuration. The links themselves are potentially a source of information concerning Earth's atmosphere depending on the transmission geometry.

The Optical Inter-orbit Communications Engineering Test Satellite (OICETS, Japanese name KIRARI) (Jono et al., 2006) was developed by the Japan Aerospace Exploration Agency (JAXA) and launched into a circular Low Earth Orbit (LEO) 23 August 2005 to an altitude of $610 \mathrm{~km}$ at an inclination of $97.8^{\circ}$. The main payload is a laser-based communication terminal called the Laser Utilizing Communication Equipment (LUCE). It was designed to conduct inter satellite laser communication experiments with the European Space Agency's (ESA) Advanced Relay and Technology MISsion (ARTEMIS) as its counterpart and orbit-to-ground communications (e.g. with the OGS, ESA's Optical Ground Station). OICETS is still in orbit and functional but the instrumentation have not been used since the conclusion of the experiments.

ARTEMIS is a multi-purpose telecommunication and technology demonstration satellite. The ARTEMIS mission began on 12 July 2001 and was subject to an unprecedented rescue that spanned 18 months after a malfunction of the upper stage of the Ariane 5 launcher. The spacecraft control team managed to raise the satellite, which was stranded in an abnormally low transfer orbit to its intended geostationary orbit (GEO) at $\sim 36000 \mathrm{~km} 21.5^{\circ}$ East on 31 January 2003 using innovative procedures and onboard advanced technology. ARTEMIS is still fully operational serving its purpose in the domains of communication, data relay and navigation.

The first ever transmission of an image by laser link from satellite-to-satellite took place on 30 November 2001 with the SILEX (Semiconductor Inter-satellite Link Experiment)

Published by Copernicus Publications on behalf of the European Geosciences Union. 
system, which consists of the OPALE (Optical Payload for Inter-Satellite Link Experiment) terminal on ARTEMIS and the PASTEL (PASsenger TELecom) terminal on the French Earth observation satellite SPOT-4 (Système Probatoire pour l'Observation de la Terre), launched on 22 March 1998, in a $832 \mathrm{~km}$ sun-synchronous LEO orbit at an inclination of $98.7^{\circ}$ (Tolker-Nielsen and Oppenhaeuser, 2002).

The link experiments between ARTEMIS and OICETS lasted in total $\sim 8$ month. The first bi-directional inter-orbit laser communication was established on 9 December 2005, experiments where carried out until the middle of August 2006 resulting in 100 successfully established links (Jono et al., 2007).

Usually the link is established well above Earth's atmosphere to ensure an optimal communication environment. For the special experiment conducted on 5 April 2006, the link was maintained until the experiment came forcibly to an end caused by atmospheric influences. The received power started to fluctuate strongly with a decrease of the average received power finally resulting in a disconnection of the link (Takayama et al., 2007).

The time of the experiment was chosen with respect to the relative positions of ARTEMIS and OICETS so the link could be established at an altitude where the residual atmosphere could be assumed as negligible. Due to the relative motion of the satellites (basically the propagation of OICETS along its orbital trajectory) a classical occultation event occurs. The laser beams start to propagate through the atmosphere at high altitudes reaching gradually denser regions as OICETS gets closer to the Earth's rim as seen from ARTEMIS. The signal can be tracked as long as the receiver at OICETS can see the transmitter at ARTEMIS (and vice versa, the link is bi-directional), thus, until OICETS sets below the horizon and the beams hit the Earth's surface. In practice, one has to consider ray bending in the atmosphere in addition to purely geometric considerations to derive the exact ray path. In fact, tracking is lost far above the surface due to fluctuations in the optical power received caused by atmospheric inhomogenities.

The results presented here are based on the data recorded at ARTEMIS. This experiment focuses on communication applications; nevertheless it can provide valuable insights for the atmospheric science community.

\section{Link experiment}

The main objective of the experiment carried out 5 April 2006 was to observe and study the atmospheric influences on the optical communication link between ARTEMIS and OICETS. The timing of the experiment was chosen in a way as to establish the bi-directional link between the two satellites in free space and subsequently observe an occultation event when the laser beams penetrated increasingly dense regions of Earth's atmosphere. The observation geometry is

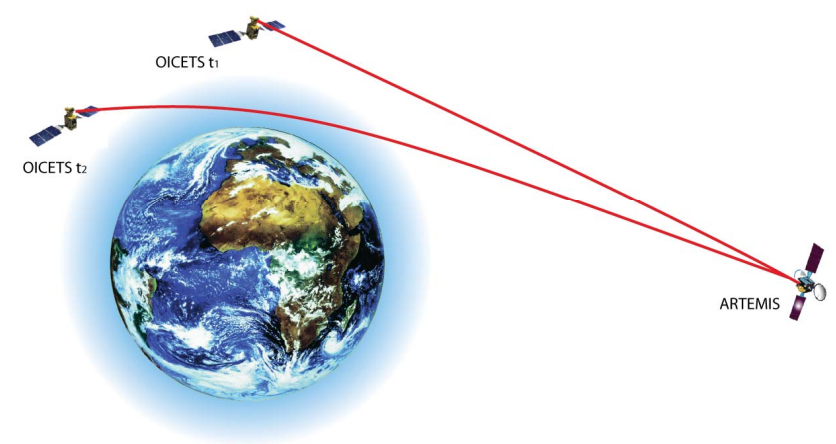

Fig. 1. Observation geometry of the communication experiment between ARTEMIS and OICETS on 5 April 2006.

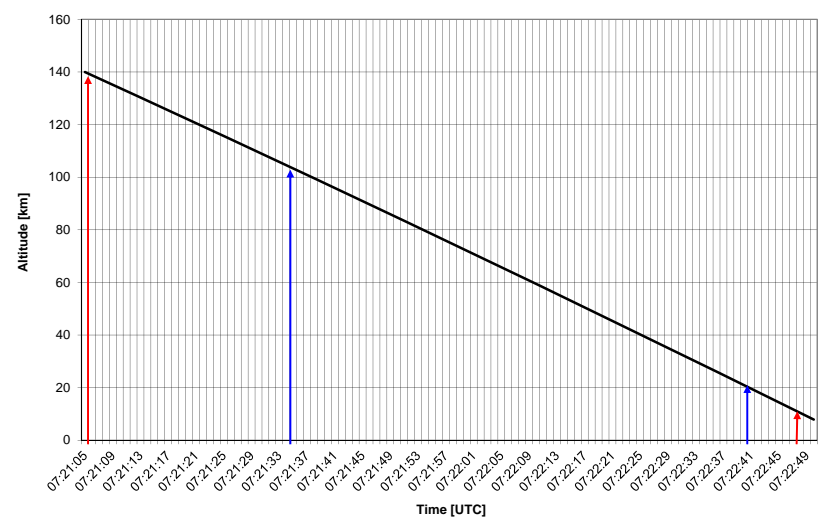

Fig. 2. Altitude - time diagram of the communication experiment between ARTEMIS and OICETS on 5 April 2006. Start and end of data records are marked in blue for ARTEMIS and red for OICETS.

illustrated in Fig. 1, showing ARTEMIS in its geostationary orbit at $\sim 36000 \mathrm{~km}$ whereas OICETS has a close to polar orbit at $\sim 610 \mathrm{~km}$. At time $t_{1}$ the bi-directional link acquisition is established and the beams propagate undisturbed trough free space. As OICETS starts to set behind Earth's atmosphere at $\mathrm{t}_{2}$ as seen from ARTEMIS, fluctuations of the received power at both detectors start to increase with a decrease of the average power received until the link is lost. The data is recorded jointly at OPALE's detector at $8 \mathrm{kHz}$ and LUCE (LUCE's sampling rate is $1 \mathrm{kHz}$ ).

The experiment started at 07:21:06 UTC and ended at 07:22:49 UTC, the resulting geometric link path altitude is shown in Fig. 2. The receiver onboard ARTEMIS lost the signal before its counterpart onboard OICETS and fluctuations of the received power (normalized by the receiver antenna area and given here in $\mathrm{nW} / \mathrm{m}^{2}$ ) are visible a bit earlier $(\sim 5 \mathrm{~s}$, which roughly corresponds to $8 \mathrm{~km})$ than in the data recorded at OICETS (Takayama et al., 2007). 


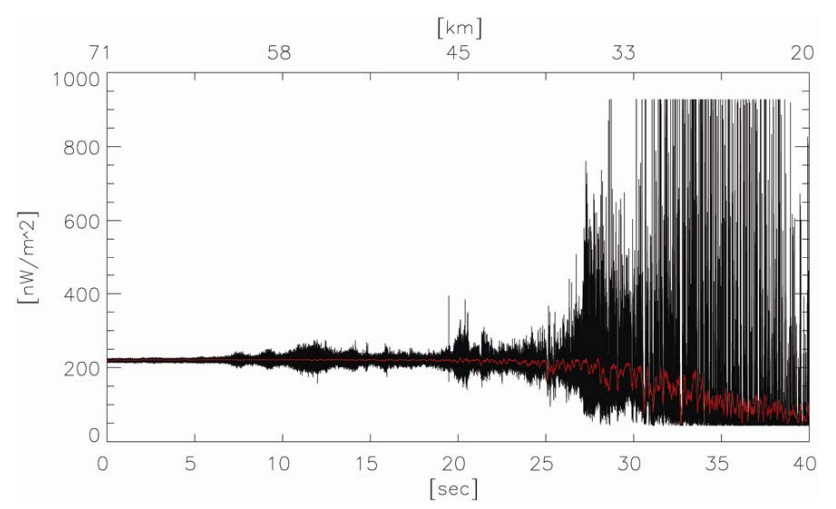

Fig. 3. Optical power detected at OPALE, 07:22:00 UTC was set to 0 in this plot, where the red line is the mean calculated from $0.1 \mathrm{~s}$ intervals.

The ARTEMIS data record starts at 07:21:36 and ends at 07:22:41. The explanation for the earlier disconnection is straightforward: although both beams propagate through the same media the signal originating from OPALE on ARTEMIS gets disturbed just before reaching the receiver on OICETS. The beam travels a long distance undisturbed through free space before being influenced by Earth's atmosphere. After emerging from the disturbing volume the distance to the receiver is rather short. The opposite is true for the beam emitted by LUCE on OICETS. The distance to the disturbing volume is rather short but after leaving Earth's atmosphere the disturbed and deflected beam has to travel a long distance which, in fact, amplifies the power fluctuations recorded at ARTEMIS. The power transmitted by LUCE is $100 \mathrm{~mW}$ which is the modulated mean value via a telescope of $0.26 \mathrm{~m}$ in diameter, the receiving telescope of OPALE has a diameter of $0.25 \mathrm{~m}$.

Figure 3 shows the power detected at ARTEMIS for a $40 \mathrm{~s}$ period starting at 07:22:00 UTC (the respective altitude range can be seen in Fig. 2) where the red line is the mean of $0.1 \mathrm{~s}$ intervals. The link is established above $100 \mathrm{~km}$ link altitude and, thus, no atmospheric influence is to be expected. The procedure is as follows: both terminals point to each other utilizing an open-loop control based on on-board orbital models. OPALE emits a strong widedivergence $(750 \mu \mathrm{rad})$ laser $(801 \mathrm{~nm})$ beam scanning a cone of uncertainty. Upon reception LUCE emits its communication beam $(847 \mathrm{~nm}, 5.5 \mu \mathrm{rad})$ towards ARTEMIS. When ARTEMIS detects the incoming communication beam from OICETS its own communication beam $(819 \mathrm{~nm})$ is switched on and the beacon is switched off. The pointing accuracy is continuously improved by the coarse and fine pointing (CPM and FPM) closed-loop systems and the bi-directional communication link is established properly.

The data is recorded with $8 \mathrm{kHz}$ by OPALE's receiver on ARTEMIS (received power, $x$ and $y$ direction angular corrections of the fine pointing mechanism). Figure 3 shows

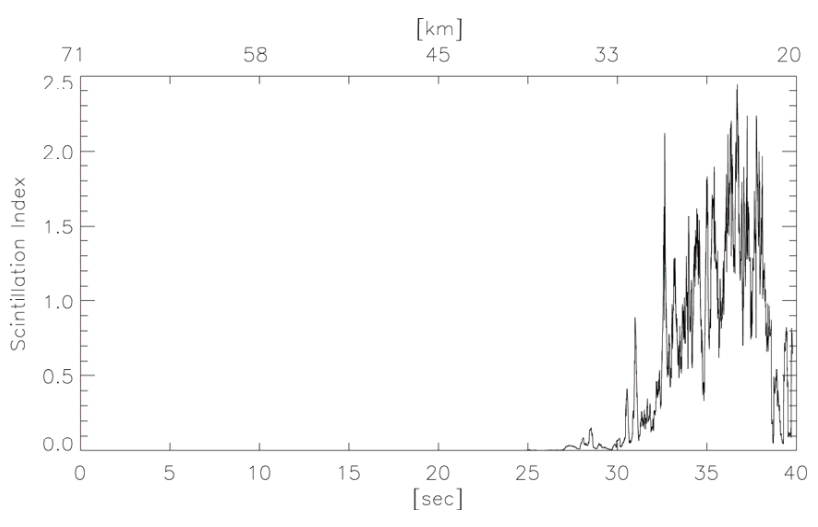

Fig. 4. Scintillation index computed from the optical power recorded at OPALE for 0.1 s intervals, 07:22:00 UTC was set to 0 in this plot.

the power received by OPALE, starting from 07:22:00 UTC, which is set to 0 in the $x$-axis of the plot. The red line is the mean power calculated at $0.1 \mathrm{~s}$ intervals. Starting from $\sim 7 \mathrm{~s}$ which corresponds to a link altitude of $\sim 66 \mathrm{~km}$ first fluctuations of the recorded power appear. The fluctuations increase until $\sim 28 \mathrm{~s}$, which corresponds to a link altitude of $\sim 36 \mathrm{~km}$, the detector also gets saturated for the first time (received power $>900 \mathrm{nW} / \mathrm{m}^{2}$ ). Starting from that point in time the power fluctuations stay at a high level saturating the detector frequently. From a brief look at Fig. 3, one might get the impression that until loss of lock enough power is received, but this is just a misleading impression caused by the power peaks recorded. The red line in Fig. 3 indicates the $0.1 \mathrm{~s}$ mean power received. It is evident that around $28 \mathrm{~s}$ the average starts to decline until it reaches very low levels at $40 \mathrm{~s}$ corresponding to a link altitude of $\sim 20 \mathrm{~km}$. The power peaks stem from the focusing effect of density inhomogenities inside the atmosphere acting like lenses on the beam.

Figure 4 shows the corresponding scintillation index Eq. (1) computed at $0.1 \mathrm{~s}$ intervals, which steeply increases around $30 \mathrm{~s}$. The same is true for the angular fluctuation of the incoming beam expressed in the angular correction performed by the fine pointing mechanism (FPM) in the $x$ - and $y$-directions (shown in Figs. 5 and 6). The $x$ and $y$-directions are referenced with respect to the FPM's coordinate system which is defined by the incoming beam representing the $z$-axis with $x$ - and $y$-axes are perpendicular to it. Thus, the geometry with respect to the Earth could only be derived knowing the exact attitude (azimuth and elevation) of the CPM. Since this data is not readily available, conclusions concerning specific horizontal and vertical effects can not be drawn. It is evident in the plots that the fine pointing mechanism also gets saturated after $30 \mathrm{~s}$ and can not compensate for the deflections of the incoming beam any more. The general pattern is similar to that of power received by the detector. The constant low level of corrections at earlier 


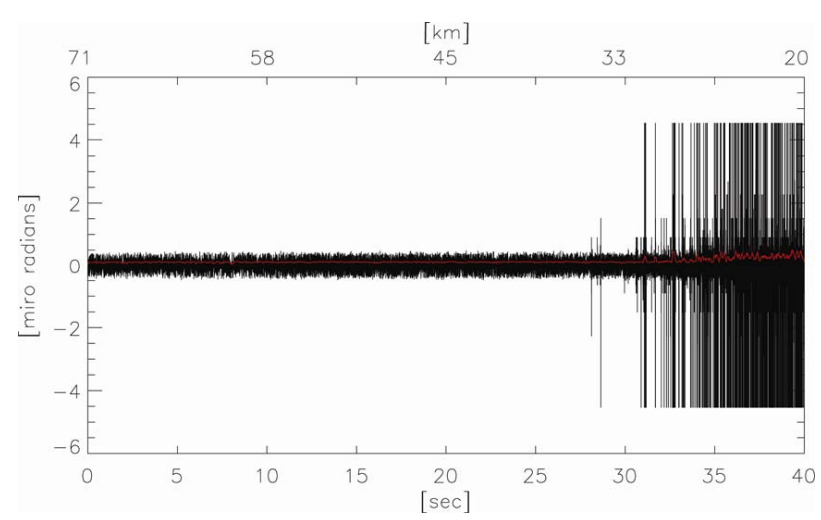

Fig. 5. Angular fluctuation of the fine pointing mechanism along the $x$-direction recorded at OPALE, 07:22:00 UTC was set to 0 in this plot. The red line is the average calculated at $0.1 \mathrm{~s}$ intervals of the corrections.

times is most likely to be attributed to combined micro vibrations of ARTEMIS and OICETS; the atmospheric effects lead immediately to strong signal variations and saturation of the mechanism. Angle of arrival errors start to increase later at the ARTEMIS side than at the OICETS side but, as mentioned, the effects are immediately strong (Perlot, 2009). A closer look at the data reveals a small gradual increase of the average corrections applied (red line in the plots) but interrupted by numerous saturation events. This behaviour seems to be caused mainly by wavefront distortions. Thus, the FPM data of ARTEMIS apparently has little useful information content with respect to atmospheric effects. The situation is potentially a little bit better on the OICETS side.

The scintillation index is computed as the variance of the flux normalized by the square of the average flux:

$\mathrm{SI}=\frac{\overline{(X-\bar{X})^{2}}}{(\bar{X})^{2}}$

The average flux is computed from a sliding mean over $0.1 \mathrm{~s}$ intervals comprising 800 detector readouts.

As mentioned earlier, Figs. 3 and 4 look similar for the power received at OICETS, with the important difference that the pronounced atmospheric effects occur a bit later and subsequently the link is disconnected at a lower altitude. This can be related to the observation geometry resulting in a shorter distance between the LEO satellite OICETS and the atmosphere compared to the distance between the GEO satellite ARTEMIS and the atmosphere. In our case (data recorded at ARTEMIS) the effects in terms of fluctuations in optical power reaching the detector are amplified by the distance (the lateral displacement caused by angular deflections increases with distance).

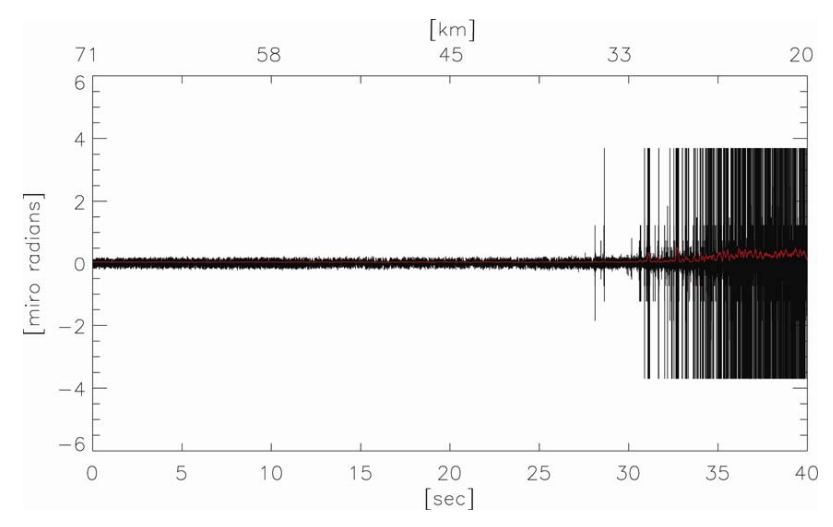

Fig. 6. Angular fluctuation of the fine pointing mechanism along the $y$-direction recorded at OPALE, 07:22:00 UTC was set to 0 in this plot. The red line is the average calculated at $0.1 \mathrm{~s}$ intervals of the corrections.

\section{Link experiment data}

Analysing the power distribution of a laser beam reaching a detector, which propagated through the atmosphere, results usually in a log-normal distribution (Churnside, 1987); above the atmosphere a normal distribution can be expected. In fact, the fluctuations of the optical power observed at the detector can be attributed to a combination of several effects in our case (Toyoshima and Araki, 2000). The atmospheric influence, beam pointing errors of the transmitter and tracking errors of the receiver (Kiasaleh, 1994), where the latter is mutually correlated since the laser communication link is a closed-loop system between the two terminals on ARTEMIS and OICETS. As can be seen in Fig. 3 (first $5 \mathrm{~s}$, beam still above atmospheric layers of significant density), the closedloop system reduces the pointing and tracking errors to a minimum, thus, fluctuations are dominated by atmospheric effects.

The first $30 \mathrm{~s}$ of the time interval displayed in Fig. 3 are analysed in more depth, since starting from second 31 the detector is frequently saturated (maximum readout $900 \mathrm{nW} / \mathrm{m}^{2}$ ) and the respective data is unreliable.

The probability density function of the received optical power can be estimated from the data observed at ARTEMIS. We process the optical power detected to obtain histograms with respect to the normalized intensity for $1 \mathrm{~s}$ periods, in total for $30 \mathrm{~s}(3 \cdot 10 \mathrm{~s})$. We use a systematic approach to determine the optimal histogram bin width as proposed in (Scott, $1979,1992)$. The bin width $w$ of those histograms is calculated as follows where $n$ denotes the sample size:

$w=2 \cdot 3^{\frac{1}{3}} \cdot \pi^{\frac{1}{6}} \cdot \sigma \cdot n^{-\frac{1}{3}}$

which is equal to:

$w=3.49 \cdot \sigma \cdot n^{-\frac{1}{3}}$ 


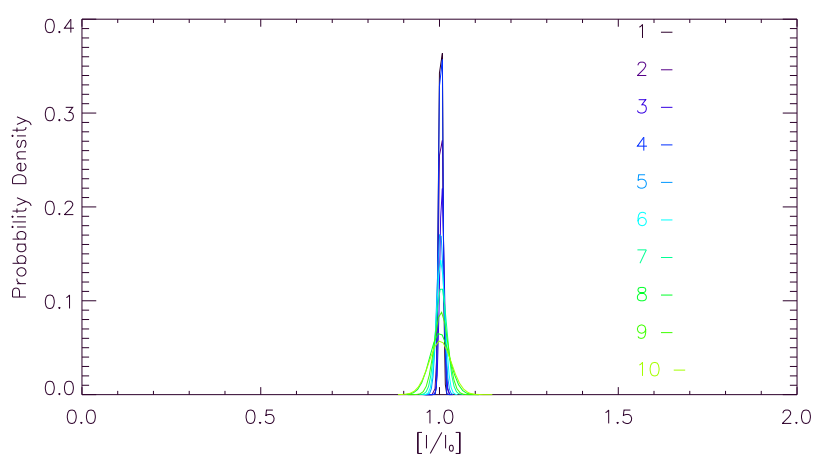

Fig. 7. The normalized intensity distribution for a period of $10 \mathrm{~s}$ starting at 07:22:00 UTC (10 times $1 \mathrm{~s}$ ).

This formulation had been derived assuming a Gaussian distribution, with the standard deviation $\sigma$. As already mentioned we can expect a log-normal distribution of the received intensity as soon as the beam starts to penetrate the atmosphere. This is the part which is of most interest with respect to turbulence. We can account for that fact by introducing the sample Skewness SF and the sample Kurtosis KF factors in calculating the bin width $w$ of the histograms. The Skewness is a measure for the asymmetry of the probability distribution of a real valued random variable and the third moment of the distribution of a random variable. The Kurtosis of the probability distribution of a real valued random variable, on the other hand, is a measure of the frequency with which extreme deviations appear, it is also denoted as the forth moment.

$\mathrm{SF}=\frac{1}{1-0.0060 \cdot \text { Skewness }+0.27 \cdot \text { Skewness }^{2}+0.0069 \cdot \text { Skewness }^{3}}$

$\mathrm{KF}=1-0.2 \cdot\left(1-e^{-0.7 \cdot \text { Kurtosis }}\right)$

To account for the real sample distribution Eq. (3) is multiplied by the Skewness factor SF Eq. (4) if the sample Skewness is $>0$ and $<3$ and by the Kurtosis factor KF Eq. (5) if the kurtosis -3 is $>0$ and $<6$.

Following this procedure 30 histograms have been constructed each covering $1 \mathrm{~s}$ of observation time, $30 \mathrm{~s}$ in total, to estimate the probability density functions from the observed data. For this analysis the optical power detected at ARTEMIS was taken starting at 07:22:00 UTC and ending $30 \mathrm{~s}$ later. To estimate the probability density functions from the histograms, a data fitting approach was used where for the fitting procedure Gaussian functions have been assumed. The intensity $I$ is normalized by the average intensity $I_{0}$ of the first second analysed. In Figs. 7 to 9 the vertical scaling changes (between max. 0.4 in Fig. 7 to max. 0.04 in Fig. 9), the areas of the underlying histograms were normalized to 1 .

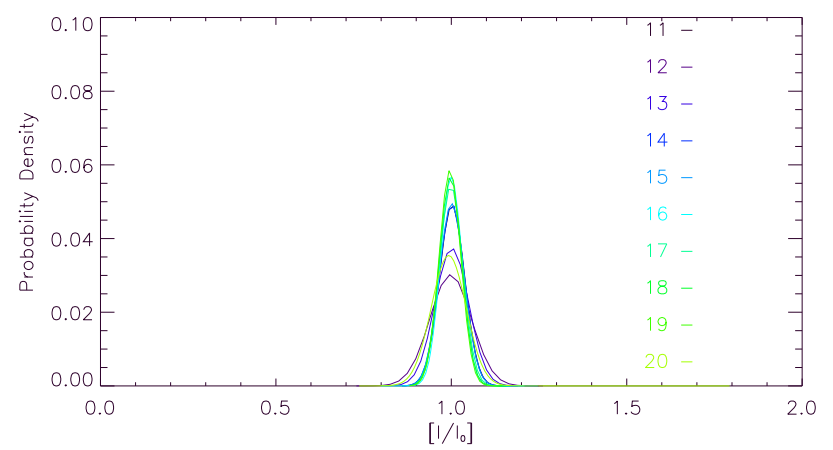

Fig. 8. The normalized intensity distribution for a period of $10 \mathrm{~s}$ starting at 07:22:10 UTC (10 times $1 \mathrm{~s})$.

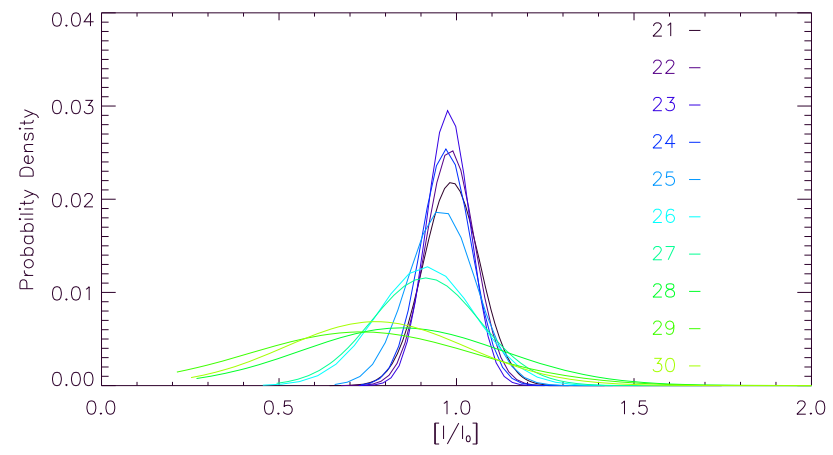

Fig. 9. The normalized intensity distribution for a period of $10 \mathrm{~s}$ starting at 07:22:20 UTC (10 times $1 \mathrm{~s})$.

It is evident that without atmospheric influence and, thus, with just pointing uncertainties caused by the relative motion of the satellites and micro-vibrations, the received power is quite stable. In Fig. 7 pronounced peaks are visible exhibiting a narrow energy distribution. This tells us that the loop system between ARTEMIS and OICETS works well. Although the distribution still stays narrow as we step forward in time during the first $10 \mathrm{~s}$ the detected optical power decreases monotonically, which indicates a monotonically increase in atmospheric density.

We see a similar picture during the next $10 \mathrm{~s}$ interval (Fig. 8) but the distributions are getting broader and the decrease is no longer monotonic. This means that the beam penetrates more turbulent atmospheric regimes resulting in density fluctuations. The density is not monotonically increasing at small scales as the beam sets through increasingly lower atmospheric layers. This becomes evident looking at second 11 which actually has the broadest distribution and the lowest power level in Fig. 8. Finally in Fig. 9, similar patterns are visible with again broader distributions and lower power levels. Around second 26 atmospheric effects strongly kick in leading to a broad distribution and, thus, quite low power levels. Although quite intense power peaks are recorded, the average power received is reduced 
dramatically. The shape of the probability density function shifts under the influence of the atmosphere from a close to Gaussian to a more log-normal distribution, as to be expected (the closed-loop tracking system minimizes effectively pointing and tracking errors). The peaks of the distributions move to the left and, thus, to lower received power.

\section{Conclusions}

The power received at the OPALE detector onboard ARTEMIS during a communication link experiment with the Japanese satellite OICETS has been analysed. The difference between this specific experiment and other optical satellite-to-satellite communication links is the fact that the link was maintained until OICETS started to set behind the atmosphere in a de facto occultation geometry. Atmospheric influences caused a disconnection of the link before the tangent point could reach the Earth's surface.

Looking at the data recorded at OICETS in the bidirectional link one recognizes that atmospheric effects appear later and the link is maintained longer than in the data recorded at ARTEMIS. This can be explained by the distance between the angular disturbance of the beam and the receiver, which is amplifying the beam displacement.

This effectively works like a "magnifying glass" for the fluctuations of the upper atmosphere, and causes earlier link loss.

Although peaks appear in the received power as the beam starts to penetrate denser layers of the atmosphere the average recorded power starts to decline until the tracking is lost.

The slight jitter visible in the $x$ (Fig. 5) and $y$ (Fig. 6) angular corrections at a constant level before atmospheric effects really kick in can be most likely attributed to micro vibrations originating onboard the satellites.

This kind of data, if available more frequently, could help to study atmospheric inhomogenities and the related scintillation phenomena with the potential to aid in validation of models.

Ongoing work concerning electromagnetic wave propagation in the Earth's atmosphere uses different parameterisations to take scintillations into account; those formulations could be improved. Work has been performed, for example, using the fast photometer data from GOMOS (Global Ozone Monitoring by Occultation of Stars) (Sofieva et al., $2009 a, b)$ for assessment of influence of scintillations on retrieval accuracy from stellar occultation experiments or from the analysis of measurements performed onboard the former Russian space-station MIR (Gurvich et al., 1996). The results are used to derive performance envelopes of different kinds of applications affected by scintillation phenomena besides using the data to address atmospheric science questions (Gurvich and Chunchuzov, 2003).
Scintillations impact many different areas like communication or remote sensing, not only in the optical domain but also in the micro wave (MW) (Gorbunov and Kirchengast, 2007). Thus, a better understanding of theses phenomena will have a positive impact on science and application.

Acknowledgements. The inter-satellite communication experiments were supported by many people through an international cooperation. The author wants to express his thanks to all members of the ARTEMIS and the OICETS teams from ESA and JAXA and Z. Sodnik (ESA) for his support.

Edited by: S. Buehler

\section{References}

Churnside, J. H. and Clifford, S. F.: Log-normal Rician probabilitydensity function of optical scintillations in the turbulent atmosphere, J. Opt. Soc. Am. A., 4, 10 October, 1923-1930, 1987.

Gorbunov, M. E. and Kirchengast, G.: Fluctuations of radio occultation signals in the $\mathrm{X} / \mathrm{K}$ band in the presence of anisotropic turbulence and differential retrieval performance, Radio Sci., 42(110), RS4025, doi:10.1029/2006RS003544, 2007.

Gurvich, A. S., Kan, V., and Fedorova, V. O.: Refraction angle fluctuations in the atmosphere from space observations of stellar scintillations, Atmos. Ocean. Phys., 31(6), 742-749, 1996.

Gurvich, A. S. and Chunchuzov, I. P.: Parameters of the fine density structure in the stratosphere obtained from spacecraft observations of stellar scintillations, J. Geophys. Res., 108, 4166-4170, 2003.

Henninger, H. and Wilfert, O.: An introduction to Free-space Optical Communications, Radioengineering, 19(2), 203-212, 2010.

Jono, T., Takayama, Y., Kura, N., Ohinata, K., Koyama, Y., Shiratama, K., Sodnik, Z., Demelenne, B., Bird, A., and Arai, K.: OICETS on-orbit laser communication experiments, Proc. of SPIE, 6105(610503), 1-11, 2006.

Jono, T., Takayama, Y., Shiratama, K., Mase, I., Demelenne, B., Sodnik, Z., Bird, A., Toyoshima, M., Kunimori, H., Giggenbach, D., Perlot, N., Knapek, M., and Arai K.: Overview of the inter-orbit and the orbit-to-ground lasercom demonstration by OICETS, Proc. of SPIE, 6457(645702), 1-10, 2007.

Kiasaleh, K.: On the probability density function of signal intensity in free-space optical communications systems impaired by pointing jitter and turbulence, Opt. Eng., 33(11), 3748-3757, 1994.

Perlot, N.: Atmospheric occultation of optical intersatellite links: coherence loss and related parameters, Appl. Opt. 48, 22902302, 2009.

Scott, W. D.: On Optimal and Data-Based Histograms, Biometrika, 66(3), 605-610, 1979.

Scott, W. D.: Multivariate Density Estimation, Theory, Practice, and Visualisation, John Wiley \& Sons, United States, ISBN:0471-54770-0, 1992.

Sofieva, V. F., Kan, V., Dalaudier, F., Kyrölä, E., Tamminen, J., Bertaux, J.-L., Hauchecorne, A., Fussen, D., and Vanhellemont, F.: Influence of scintillation on quality of ozone monitoring by GOMOS, Atmos. Chem. Phys., 9, 9197-9207, doi:10.5194/acp9-9197-2009, 2009. 
Sofieva, V. F., Dalaudier, F., Kan, V., and Gurvich, A. S.: Technical note: Scintillations of the double star a Cru observed by GOMOS/Envisat, Atmos. Chem. Phys., 9, 8967-8973, doi:10.5194/acp-9-8967-2009, 2009.

Takayama, Y., Jono, T., Koyama, Y., Kura, N., Shiratama, K., Demelenne, B., Sodnik, Z., Bird, A., and Arai, K.: Observation of atmospheric influence on OICETS inter-orbit laser communication demonstrations, Proc. of SPIE, 6709, 67091B, 1-9, doi:10.1117/12.736789, 2007.
Tolker-Nielsen, T. and Oppenhaeuser, G.: In Orbit test result of an Operational Optical Intersatellite Link between ARTEMIS and SPOT4, SILEX, Proc. of SPIE 4635, 1-15, 2002.

Toyoshima, M. and Araki, K.: Effects of time averaging on optical scintillation in a ground-to- satellite atmospheric propagation, Appl. Opt., 39(12), 1911-1919, 2000. 